\title{
Olive Oil Ripping Time Prediction Model based on Image Processing and Neural Network
}

\author{
Mutasem Shabeb Alkhasawneh \\ Software Engineering Department, Faculty of Information and Technology \\ Ajloun National University, P.O. Box 43, Ajloun 26810, Jordan
}

\begin{abstract}
The agriculture sector in Jordan depends very much on planting the olive trees. More than ten million of olive trees are planted in the Jordanian soil. Olive fruit are harvested for two purposes; either to produce oil or to produce olive table (pickled olive). Olive fruit harvesting time for extracting the oil from the olive fruit is crucial. Hence, harvesting the olive fruit on ripping time gives the best amount and quality of oil. It also, could lose $15 \%$ to $20 \%$ of multiple values because of harvesting time. Olive fruit ripping time is varied since it depends on the rainfall, temperature and cultivation. A system to predict the optimal time for harvesting olive fruit for producing oil only is introduced. It based one Digital Image Processing (DIP) and artificial intelligent neural network. Moreover, four features were extracted from the olive fruit image based on the red, green and blue colors. The proposed system tested olive fruits in three stages of ripping time; under ripping, on ripping and over ripping. The classification accuracy achieved in the three stages was $97.51 \%$ in under ripping stage $95.10 \%$ in ripping stage, and $96.12 \%$ in over ripping stage. The proposed system performance was $96.14 \%$.
\end{abstract}

Keywords-Neural network; image processing; olive ripping time; prediction; classifications

\section{INTRODUCTION}

Olive tree is the most important tree in Jordan. It represents $70 \%$ of the fruit tree in the country [1]. Approximately 560,000 dunums $(560,000,000 \mathrm{~m} 2)$ are planted with more than 10.5 million of olive trees, which represent $72.00 \%$ of the agricultures land in the kingdom of Jordan [2]. There are three common kinds of olive trees in Jordan; Nabali olive with high oil content between $28 \%$ and $33 \%$. Rasi olive with percentage of oil varies from $15 \%$ to $28 \%$ and Sori olive for olive table. Furthermore, the investment in the olive sector passed one Billion Jordanian Dinar (JD) in 2018. Also more than 80,000 Jordanian families have benefit from this sector. According to an Agriculture Ministry of Jordan, the olive oil production increases from year to year, where 24,000 tons of olive oil were produced in 2018 with average price varied between $134,880,000 \mathrm{JD}$ to $142,500,000 \mathrm{JD}$ depending on the amount of produced oil in the season and the type of the olive oil. The amount and the quality of olive oil depend on rainfalls, cultivation and the harvesting time. By harvesting the olive trees on the ripping time, the best amount and quality of olive oil can be achieved [3]. Jordan loses $15 \%$ to $20 \%$ of the amount of olive oil because of harvesting time. By either harvesting the olive fruits before or after the suitable harvesting time [2]. The olive oil characteristics have its own rank among all kinds of oils consumed by human being because of its pleasant flavor, plat ability, stability and health benefits. Harvesting the olive tree before or after ripping time, reflects the quality grade of oil characteristics, oxidation, stability and nasturtium value of the obtained product [4]. The majority of olive oil (94\%) does not have the best commercial quality, because the olive fruit is not picked on the optimal harvesting time [5]. According to [6] harvesting olive fruits must be done when the oil reaches the best quality and the highest amount of oil level [3, 7]. In the Harvesting olive fruit season, the farmers attempt to estimate ripping time based on the color, texture, size and shape of the fruits. This estimation can be done by walking through the olive tree field. Factors such as the size and the shape varied depending on the amount of the rainfall, dryness and cultivation during the year. Also, factors like the color and texture of the fruit are subjective and depend on the farmer experiences. Therefore, farmers need to have additional opinion to determine the harvesting time precisely. As an additional authenticates opinion researchers have proposed different methods like analytical static [7] and intelligent system based on Digital Image Processing (DIP) and Artificial Intelligent (AI) and indeed Artificial Neural Networks (ANN). For instance, in the medical field DIP and ANN were used to classify image breast cancer and detecting brain tumor [8, 9] also new model of neural network was proposed to classify six types of disease; the proposed model has shown very high performance. A convolutional neural network was used in face image manipulation detection [10, 11]. DIP and ANN have shown great potential in managing road traffic system as in [12-14]. In addition, ANN have been widely used to predict the landslide hazard map [15]. Moreover, as a tool of classification ANN and DIP have been widely used in different fields as mentioned in [16-20]. This paper presents a model to determine the optimal time for harvesting olive fruits for oil. The new model relies on Digital Image Processing and Neural Network.

In this section, the aim of this study and general back ground for the proposed system was introduced. In Section 2, the related previous work that used Image Processing and ANN is summarized. Section 3 the data collection and the structure of Cascade Forward Neural Network (CFNN) were explained. Section 4, Image Preprocessing, Section 5, Feature Extraction, Section 6, Classification Model, Section 7, Experimental Methodology, Section 8, Results and discussions and Conclusion in Section 9.

\section{RELATED WORKS}

Maturities, ripeness, quality and diseases of the fruits have been subject of interest for many studies and included 
different types of fruits such as Grape, Apple, Tomatoes, Cucumber, Orange, Palm oil fruits and many other fruits [2125]. Studies on Olive fruit can be divided into classification the quality of olive fruits for olive table (pickled olive) [26, 27], external damage and diseases of olive fruit [28, 29] and olive fruit ripping time for olive oil [30-32].

To determine the stage of maturity of the olive fruit for oil, various methods were applied [29]. Maturity index (MI) is the common traditional used method to determine the optimal time for harvesting olive fruits for oil. To determine the MI, one KG of olive fruit must be collected randomly from several trees with same verity. Then, 100 olives fruit picked up randomly from $1 \mathrm{KG}$. Each 100 fruit sample separated into different eight categories of color starting from " 0 " deep green to "7" purple or black color. Multiply the number of fruits in each color category by the number of that color category ( 0 to 7) and add up all the numbers and the result divide by 100 . When the MI value is 5 it's the optimum time to harvest the olives. The MI method is not accurate since it depends on the human judgment. The work in [33] approves the machine more accurate than the human on the classifying olive table. Thus, finding an automatic system to determine the optimal harvesting olive fruits time has become subject of interest for many studies. In this section, the automatic system based on DIP and ANN will be reviewed.

An automatic model for Ripping Index (RI) based on DIP and ANN introduced by [30]. One KG of olive fruits samples were collected randomly and classified to three classes, then analyzed to measure some chemical parameters; oil, sugar and total phenolic content in the olive fruits. The chemical parameters were compared with color of the fruits. The three chemical inputs and the fruit color were used as input to Multi-Layer Perceptron. The results prove that the proposed system has better results than the traditional used RI.

A study introduced on Arbquina olive fruit type. It aimed to classify the maturity of the olive fruits based on changing the color during the fruits maturity stages; green, yellow green, turning, purple, and black. The study highly recommended using the color of olive fruit as maturity index [34].

A color scale was used to determine the maturity of olive and grape fruits. The color scale generated using the wellknown support vector machine based on the multidimensional regression. 250 and 200 samples respectively of grape and olive fruit were used for experimental purposes. The developed color scale can be used in various phenolic states on the fruit [21].

Image analysis was used to determine the maturity of the olive fruits. The system relied on used image segmentations include edge detections and the histogram values for the red, green and blue colors values were calculated. The olive fruit was classified to five categories based on fruit skin color; bright green, greenish yellow, greenish yellow with red black or reddish spots greater than reddish-brown, greenish-yellow with black and/or reddish spots less than reddish-brown and $100 \%$ blackish-purple or black [35].
Olive ripping phase was estimated to six phases based on the fruit skin color. The skin color histogram was manipulated in the feature selection phase, and Multi-Layer Perceptron neural network as a classifier. The obtained results showed promising results [36].

A technic was proposed to determine the olive oil based on olive fruit ripening stage using the DIP. The method start by acquiring the olive fruit image then image processing features extraction, features selection and finally the regression mode for classification the oil quality [37].

The quality of the olive oil strongly related to the quality of the collected fruits. A mathematical model based on partial least squares Discriminant Analysis (PLD), and image processing with neural network introduced to classify four type of olive fruits (Mission, Manzanillo, Ascolano, and Sevillan) to four grades (unacceptable, borderline, acceptable, and optimal). $75 \%$ and $70 \%$ correct classification rates offered by PLD , whereas $93.0 \%$ and $90.0 \%$ classification rate offered by DIP and neural network [38]. Classifying the olive fruit into different categories are done manually before the oil extraction process. Therefore, an online system that automatically cans categories the olive fruits to their quality level in different class. The study, focused on the olive fruits picked up from the tree or from the ground. In this research, the online system constructed of DIP (image entropy, grey level co-occurrence matrix and statistics like Contrast, Correlation, Energy and Homogeneity) and for classification ANN and Support Vector Machine (SVM) with achieved calcification accuracy $98.8 \%$ and $98.4 \%$ for the former and later respectively [31].

\section{Methodology}

The methodology of this research can be divided into: collecting olive fruit images, image processing, features extraction and classification as shown in Fig. 1.

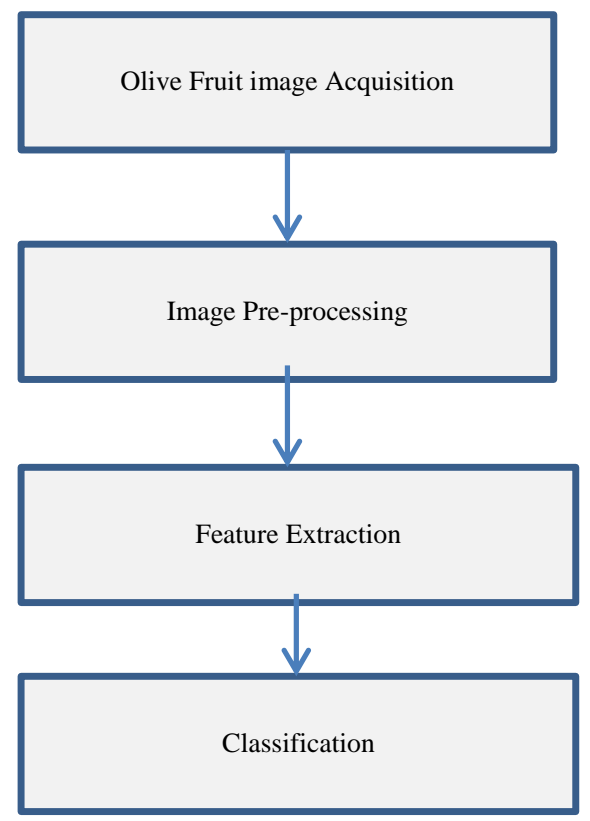

Fig. 1. Flow Chart of the Work Methodology. 


\section{A. Olive Samples}

The data of the olive fruit were collected during the season 2010 from Rasi olive tree farm in Hawara, Irbid, Jordan. The data were collected between August and December olive fruit color, size and shape change faster than rest of the year. Therefore Olive fruit was randomly picked up and images were taken.

Fig. 2 shows the differences between the olive fruit color between August and December. High temperature and dry weather speed up the change of olive fruit color and vice versa, as mentioned the Sori olive is the subject for this study.

\section{B. Acquisition of Images}

Olive fruit images were taken at the farm, the images where taken in day light. Fifty images were taken every week from August to December with totally 1200 images, only ten suitable images were chosen for further process with totally 240 images. The Image acquisition system consists of digital camera Canon EOS 7D, 18 Megapixels, and white sheet of paper was setup in the farm. The deformable durable material was chosen in an attempt to approximate the type that would be available in regular farmers' hands.

1) Image pre-processing: In order to have a proper image for extracting information, all images must be with a standard size; thus, olive image were scaled downed to $50 \%$ of its original size using Image Processing Tool Box in MATLAB Released 2013a. Then, filtering the image and remove any noise. Finally, removing the white back ground from the image, in order to have only the olive fruit as shown in Fig. 3.
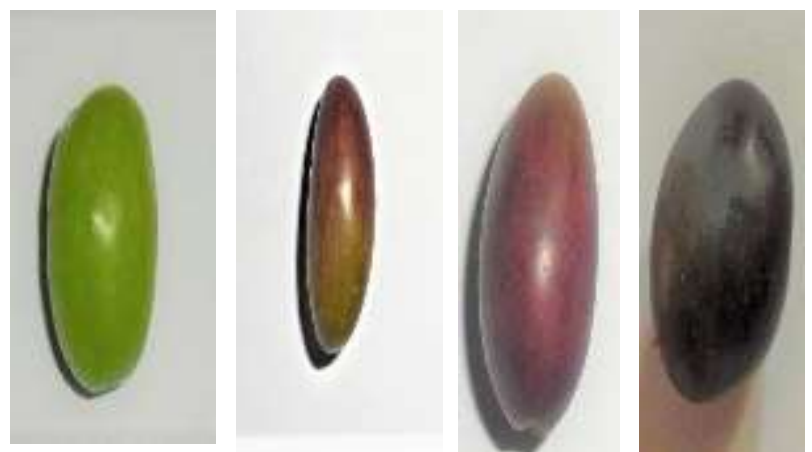

(a) From August (b)September (c) November (d) December

Fig. 2. Olive Fruit Color between August and December.

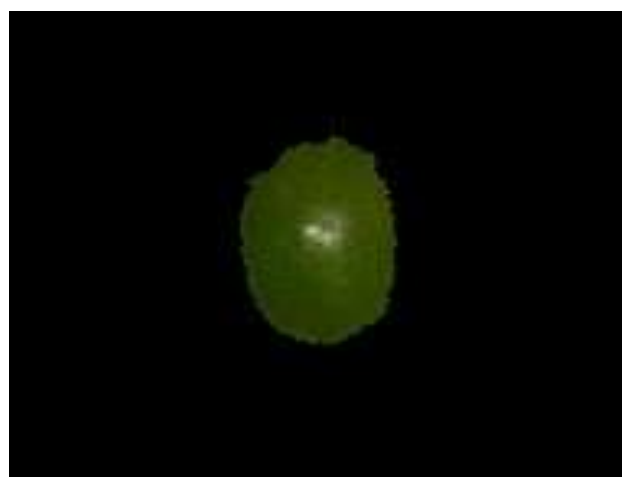

Fig. 3. Olive Fruit after Removing Background.
2) Feature extraction: The aim of feature extraction is to achieve some features, which can be used in the classification system. In most cases the color of the fruit is the strongest indication for the fruit maturity. The color of the olive fruits starts changing gradually from dark green to dark black. The image color component Red, Green and Blue (RGB) was used to calculate the mean, median and Standard Deviation (SD) of RGB colors.

\section{Classifications Model}

Cascade forward neural network (CFNN) is one of the artificial neural network types. It construct of input, hidden and output layer. Where, input layer connected to one or more hidden layer which also connected to output layer. CFNN also has a connection between the input layer and every layer in the neural network. In addition CFNN also has another connection from each layer to the successive layers in the network Demuth [39]. CFNN has the same principle of work and methodology that multi-layer feed forward neural network do, but the extra two connections [40]. The later connections are introduced to improve the neural network generalizations [41]. Fig. 4. Shows topology of CFNN with three layers; input, hidden and output layer.

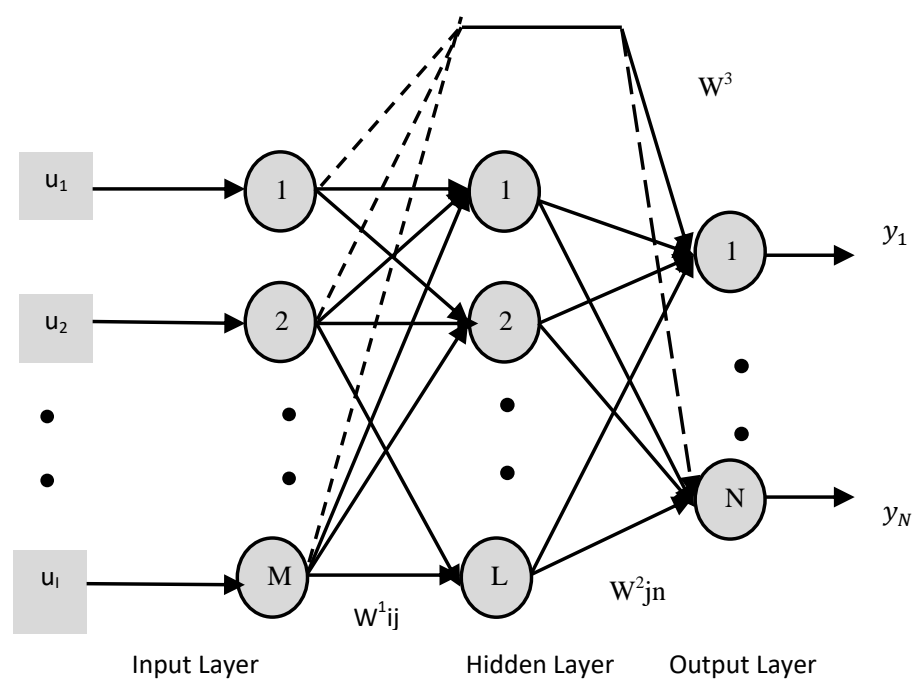

Fig. 4. Cascade Forward Neural Network.

Each neuron in the hidden layer receives input from the input layer. The total input to the hidden layer from all input neurons can be found as in Eq. (1).

net $_{h_{j}^{(k)}}=\sum_{i=1}^{M} w_{i, j}^{1} u_{i}^{(k)}$ for $1 \leq j \leq M$

$n e t_{-} h_{j}$ is the total of input weights to the hidden layer. where $i=1,2, \ldots, M$, indexed to the input layer neurons. $\mathrm{W}_{\mathrm{i}, \mathrm{j}}^{1}$ represent the weight from input layer neuron to hidden layer. $k$ denote to the moment. $u_{i}^{(k)}$ represent the inputs of neural network.

Eq. (2) shows the total output of the hidden layer at time $k$ $o_{-} h_{j}^{(k)}$. Where $f(*)$ is the linear or nonlinear of hidden layer output function. 
$o_{-} h_{j}^{(k)}=f\left(\operatorname{net}_{h_{j}^{(k)}}\right)$

The output of one hidden layer of CFNN can be found as in Eq. (3)

$y_{n}^{(k)}=g\left(\sum_{j=1}^{L} w^{2}{ }_{j n} o_{h_{j}^{(k)}}+\sum_{i=1}^{M} w^{3}{ }_{i, n} u_{i}^{k}\right)$ for $1 \leq n \leq N$

Where, $y_{n}^{(k)}$ is the outputs of CFNN, and $n=1,2, \ldots, \mathrm{N}$. is the output neurons.

$g(*)$ is the linear or nonlinear output function of output layer. The connection weight between the nodes in the hidden layer and output layer represented by $\mathrm{W}^{2}{ }_{\mathrm{jn}}$.

\section{EXPERIMENT METHODOLOGY}

The experimental process was divided into; preparing the data that will be used for CFNN, the structures of the CFNN and the CFNN performance measurement. Preparing the olive fruit data set includes: normalizing all the features that extracted from olive fruit such RGB color. The later has three different values namely mean, mean Standard Deviation (SD), median and median SD. For each feature the normalization can be varied between 0 and 1 based on the Eq. (7).

Normalised value $(i)=\frac{\text { value }(i)-\text { minimum value }(I)}{\text { maximum value }(I)-\text { minimum value }(I)}$

Where the value $(i)$ is the value to be normalized and $(I)$ is the minimum or the maximum pixels value for every single feature. The intelligent system targets are divided into three classes; before ripping time, on ripping time and over ripping time are represented by 0,1 and 2, respectively. Moreover, dividing the normalized data before entering the CFNN is a crucial to avoid over fitting during the training process of the CFNN therefore; the normalized data was randomized and then divided to three different sets, $70 \%, 15 \%$ and $15 \%$ for training, testing and validation, respectively.

As mentioned, the CFNN with one input, one hidden and output layer structure can solve most of the classifications problems. The number of the neurons in the hidden layer has a high impact on the CFNN performance. For this research, the number of the hidden neurons was tested from 5 to 50 neurons, by increasing the number of the neurons by 5 each time and the accuracy was measured. CFNN with 9 neuron in the hidden layer was found to be the optimal for this study. CFNN Over fitting is another common errors that facing neural network during the training. To overcome this problem, the neural network will stop after 500 epoch, If the performance the neural network could not reach the 0.01 as Mean Square Error (MSE).

The performance of the CFNN classification accuracy was measured during the training and testing stage. The accuracy was calculated by using the Eq. (8).

Accuracy $=\frac{\text { Number of olive fruit ripping satge correctlty classfied }}{\text { Total number of olive fruit sample }}$

\section{RESUlTS AND DiscussiOnS}

In this section and following subsections, the feature extractions and CFNN performance for the olive fruit maturity stages; under ripping time, on ripping time and over ripping time will be evaluated.

\section{A. Under Ripping Stage}

The olive fruit color, in under ripping stage, varied between the dark green and yellowish. In this stage the average space of the red color was $66.83 \%$ and the Mean SD was $2.56 \%$, while the median and SD median for the red color was $66.71 \%$ and $4.50 \%$ respectively. The values for green color were $72.30 \%, 2.08 \%$ and $72.15 \%, 1.50 \%$ for the mean, SD mean, median and SD median respectively. Moreover, $1.50 \%, 40.78 \%, 3.01 \%, 39.8 \%$ and $2.82 \%$ were in orders the values of mean blue, means SD, median blue and median SD blue value. For this stage the red color is considered green color [42] as shown in Table I.

Fig. 5 shows the CFNN classification accuracy for training and testing. The results show that the CFNN classified the olive fruit on under ripping stage with $99.10 \%$ for training data and $97.51 \%$ for testing data.

TABLE I. FEATURES EXTRACTION VALUES FOR UNDER RIPPING STAGE

\begin{tabular}{|l|l|}
\hline Under Ripping Stage & $66.83 \%$ \\
\hline Mean Red Value & $2.56 \%$ \\
\hline Mean SD Red Value & $66.71 \%$ \\
\hline Median Red Value & $4.5 \%$ \\
\hline Median SD Red value & $72.3 \%$ \\
\hline Mean Green Value & $2.08 \%$ \\
\hline Mean SD Green Value & 72.15 \\
\hline Median Green Value & $1.5 \%$ \\
\hline Median SD Green Value & $40.78 \%$ \\
\hline Mean Blue Value & $3.01 \%$ \\
\hline Mean SD Blue Value & $39.8 \%$ \\
\hline Median Blue Value & $2.82 \%$ \\
\hline Median SD Blue Value & \\
\hline
\end{tabular}

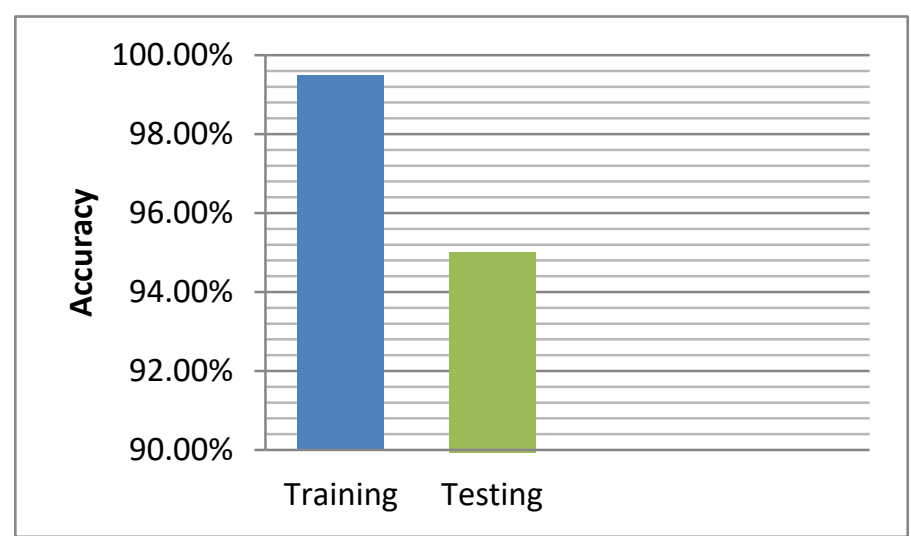

Fig. 5. CFNN Training and Testing Results for under Ripping Olive Fruits Data Set. 


\section{B. On Ripping Stage}

The olive fruit color on ripping stage is varied between blackish to purple. The red color values for the mean, mean $\mathrm{SD}$, median and median SD in were in ordered $59.71 \%, 2.5 \%$, $60.12 \%$ and $3.08 \%$. The average space of the green was $40.20 \%$ and the Mean SD was $1.90 \%$, while the median and SD median for the red color was $38.99 \%$ and $2.21 \%$ respectively. The values for blue color were $35.90 \%, 3.56 \%$ and $36.79 \%, 3.31 \%$ for the mean, SD mean, median and SD median, respectively as shown in Table II.

$98.58 \%$ of the olive was correctly classified for training and $95.10 \%$ of olive fruits were correctly classified in the testing stage as can be noticed from Fig. 6.

\section{Over Ripping Stage}

The olive fruit color for over ripping stage is dark black. The values of red, green and blue color as seen from Table III were Mean red 23.65\%, mean SD 1.67\%, Median red $23.63 \%$ and median SD $1.71 \%$. The green color values for the mean, mean SD, median and median SD in were in ordered $21.8 \%$, $1.34 \%, 21.90 \%$ and $1.33 \%$. The values for blue color were $22.23 \%, 1.11 \%$ and $21.97 \%, 2.10 \%$ for the mean, SD mean, median and SD median respectively as shown in Table III.

TABLE II. FEATURES EXTRACTION VALUES FOR ON RiPPING STAGE

\begin{tabular}{|l|l|}
\hline Under Ripping Stage & $59.71 \%$ \\
\hline Mean Red Value & $2.5 \%$ \\
\hline Mean SD Red Value & $60.12 \%$ \\
\hline Median Red Value & $3.08 \%$ \\
\hline Median SD Red Value & $40.20 \%$ \\
\hline Mean Green Value & $1.90 \%$ \\
\hline Mean SD Green Value & $38.99 \%$ \\
\hline Median Green Value & $2.21 \%$ \\
\hline Median SD Green Value & $35.90 \%$ \\
\hline Mean Blue Value & $3.56 \%$ \\
\hline Mean SD Blue Value & $36.79 \%$ \\
\hline Median Blue Value & $3.31 \%$ \\
\hline Median SD Blue Value & \\
\hline
\end{tabular}

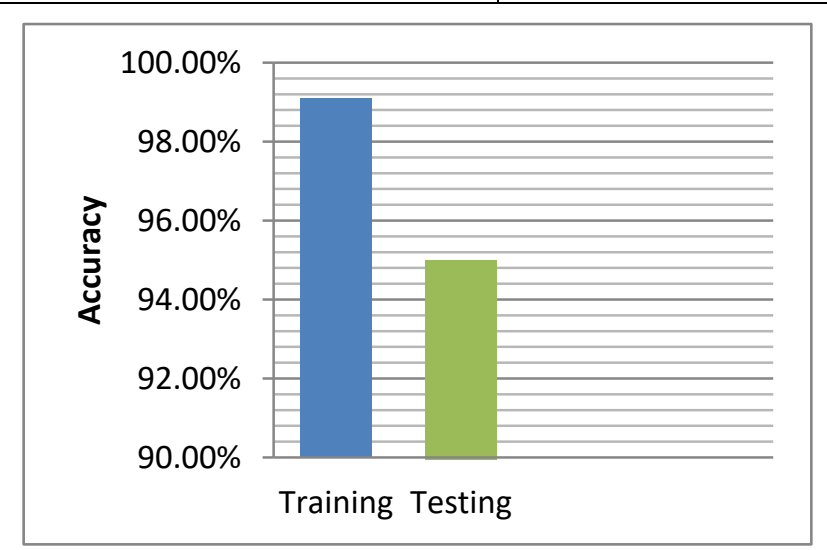

Fig. 6. CFNN Training and Testing Results for on Ripping Olive Fruits Data Set.
TABLE III. FEATURES EXTRACTION VALUES FOR OVER RIPPING STAGE

\begin{tabular}{|l|l|}
\hline Under Ripping Stage & $23.65 \%$ \\
\hline Mean Red Value & $1.67 \%$ \\
\hline Mean SD Red Value & $23.63 \%$ \\
\hline Median Red Value & $1.71 \%$ \\
\hline Median Red Value & $21.8 \%$ \\
\hline Mean Green Value & $1.34 \%$ \\
\hline Mean SD Green Value & $21.90 \%$ \\
\hline Median Green Value & $1.33 \%$ \\
\hline Median SD Green Value & $22.23 \%$ \\
\hline Mean Blue Value & $1.11 \%$ \\
\hline Mean SD Blue Value & $21.97 \%$ \\
\hline Median Blue Value & $2.10 \%$ \\
\hline Median SD Blue Value & \\
\hline
\end{tabular}

In Fig. 7, it can be clearly seen that the CFNN achieved $97.00 \%$ of accuracy through the training stage and $96.12 \%$ accuracy through the testing stage.

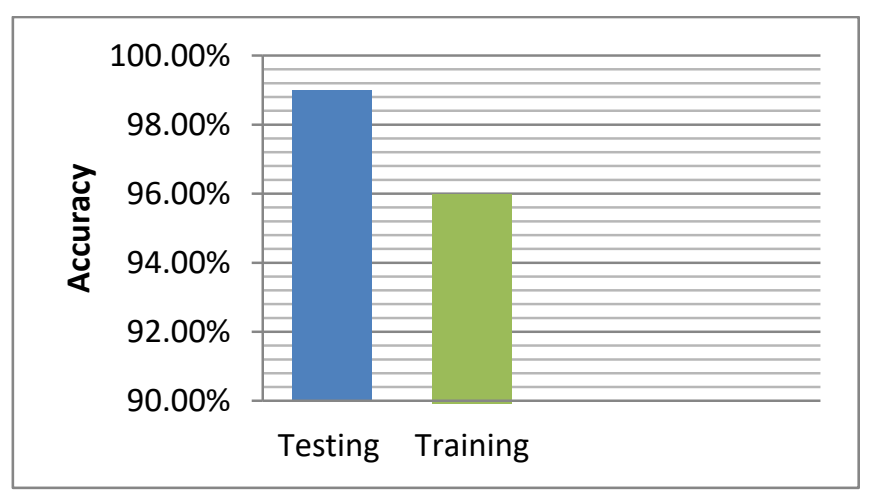

Fig. 7. CFNN Training and Testing Results for under Ripping Olive Fruits Data Set.

\section{CONCLUSION}

This work introduced a system to predict the optimal time for harvesting olive fruits for extracting oil. The methodology of this work was started by taking pictures for the Rasi olive in different period of time. Then extracting features from olive fruits RGB color. CFNN neural network was used to classify the olive fruits to three levels; under ripe, on ripe and over ripe. In this study an artificial intelligent system was introduced for decision support task of harvesting olive fruits for extracting oil, where the amount and the quality of the olive are increased. Future work should be apple to predict the olive ripping time in the real time.

\section{REFERENCES}

[1] Al Rwahnih, M. and M. Al Khasawneh, Jordan, in Production and exchange of virus-free plant propagating material in the Mediterranean region, B. Di Terlizzi, A. Myrta, and V. Savino, Editors. 2001, Bari : CIHEAM. p. 83-85.

[2] Times, J., Olive trees cover $72 \%$ of Jordan's agricultural land. Jordan times, 2019.

[3] Zamora, R., M. Alaiz, and F.J. Hidalgo, Influence of cultivar and fruit ripening on olive (Olea europaea) fruit protein content, composition, and 
antioxidant activity. Journal of agricultural and food chemistry, 2001. 49(9): p. 4267-4270. DOI: 10.1021/jf0104634.

[4] Gasparini, P., Extra Virgin Olive Oil Production in Jordan.Preliminary overlook on the sector. United nations industrial development organization., 2001. 1(1).

[5] Liphschitz, N., et al., Wild Olive (Olea europaea) Stones from a Chalcolithic Cave at Shoham, Israel and their Implications. Tel Aviv: Journal of the Institute of Archaeology of Tel Aviv University, 1996. 23: p. 135-142. DOI: 10.1179/033443596788138729.

[6] Stefanoudaki, E., F. Kotsifaki, and A. Koutsaftakis, Classification of virgin olive oils of the two major cretan cultivars based on their fatty acid composition. Journal of the American Oil Chemists' Society, 1999. 76(5): p. 623-626. doi:10.1007/s11746-999-0013-7.

[7] García, J.M., S. Seller, and M.C. Pérez-Camino, Influence of Fruit Ripening on Olive Oil Quality. Journal of Agricultural and Food Chemistry, 1996. 44(11): p. 3516-3520. doi.org/10.1021/jf950585u.

[8] Abdul-Kadir, N.A., et al. Applications of cascade-forward neural networks for nasal, lateral and trill arabic phonemes. in Information Science and Digital Content Technology (ICIDT), 2012 8th International Conference on. 2012.

[9] S. Giraddi and S. V. Vaishnavi, "Detection of Brain Tumor using Image Classification," 2017 International Conference on Current Trends in Computer, Electrical, Electronics and Communication (CTCEEC), Mysore, 2017, pp. 640-644, doi: 10.1109/CTCEEC.2017.8454968.

[10] L. Minh Dang, Syed Ibrahim Hassan, Suhyeon Im, Hyeonjoon Moon, Face image manipulation detection based on a convolutional neural network,Expert Systems with Applications, Volume 129,2019,Pages 156-168,ISSN 09574174, https://doi.org/10.1016/j.eswa.2019.04.005.

[11] Guanjun Guo, Hanzi Wang, Yan Yan, Jin Zheng, Bo Li, A fast face detection method via convolutional neural network, Neurocomputing, Volume 395, 2020, Pages 128-137, ISSN 0925-2312, https://doi.org/10.1016/j.neucom.2018.02.110.

[12] Zou, X., Y. Fu, and X. Li. Image Feature Recognition of Railway Truck Based on Machine Learning. in 2019 IEEE 3rd Information Technology, Networking, Electronic and Automation Control Conference (ITNEC). 2019.

[13] Jagadamba, G., S. Purohit, and G. Chayashree. Campus Vehicle Monitoring Through Image Processing. in Emerging Research in Electronics, Computer Science and Technology. 2019. Singapore: Springer Singapore.

[14] Barreto S.C., Lambert J.A., de Barros Vidal F. (2019) Using Synthetic Images for Deep Learning Recognition Process on Automatic License Plate Recognition. In: Carrasco-Ochoa J., Martínez-Trinidad J., OlveraLópez J., Salas J. (eds) Pattern Recognition. MCPR 2019. Lecture Notes in Computer Science, vol 11524. Springer, Cham. https://doi.org/10.1007/978-3-030-21077-9_11.

[15] Alkhasawneh, M.S., et al., Intelligent Landslide System Based on Discriminant Analysis and Cascade-Forward Back-Propagation Network. Arabian Journal for Science and Engineering, 2014. 39(7): p. 5575-5584. 10.1007/s13369-014-1105-8.

[16] L. Benali, G. Notton, A. Fouilloy, C. Voyant, R. Dizene, Solar radiation forecasting using artificial neural network and random forest methods: Application to normal beam, horizontal diffuse and global components, Renewable Energy, Volume 132, 2019, Pages 871-884, ISSN 09601481, https://doi.org/10.1016/j.renene.2018.08.044.

[17] $\mathrm{Li}$, D., et al., A novel CNN based security guaranteed image watermarking generation scenario for smart city applications. $\begin{array}{lllll}\text { Information Sciences, } 2019 . & 479: & \text { p. } & 432-447 .\end{array}$ doi.org/10.1016/j.ins.2018.02.060.

[18] Kelsey A. Ramírez-Gutiérrez, Alejandro Medina-Santiago, Alfonso Martínez-Cruz, Ignacio Algredo-Badillo, Hayde Peregrina-Barreto, RETRACTED: Eggshell deformation detection applying computer vision, Computers and Electronics in Agriculture, Volume 158, 2019,Pages 133-139, $\quad$ ISSN 1699,https://doi.org/10.1016/j.compag.2019.01.036.

[19] Chang, Y., Research on de-motion blur image processing based on deep learning. Journal of Visual Communication and Image Representation, 2019. 60: p. 371-379. doi.org/10.1016/j.jvcir.2019.02.030.
[20] Abdollahi, H., et al., Prediction and optimization studies for bioleaching of molybdenite concentrate using artificial neural networks and genetic algorithm. Minerals Engineering, 2019. 130: p. 24-35. doi.org/10.1016/j.mineng.2018.10.008.

[21] Avila, F., et al., A method to construct fruit maturity color scales based on support machines for regression: Application to olives and grape seeds. Journal of Food Engineering, 2015. 162: p. 9-17. doi.org/10.1016/j.jfoodeng.2015.03.035.

[22] Yuri, J.A., et al., Effect of cultivar, rootstock, and growing conditions on fruit maturity and postharvest quality as part of a six-year apple trial in Chile. Scientia Horticulturae, 2019. 253: p. 70-79. doi.org/10.1016/j.scienta.2019.04.020.

[23] Pacheco, W.D.N. and F.R.J. López. Tomato classification according to organoleptic maturity (coloration) using machine learning algorithms KNN, MLP, and K-Means Clustering. in 2019 XXII Symposium on Image, Signal Processing and Artificial Vision (STSIVA). 2019. doi: 10.1109/STSIVA.2019.8730232.

[24] Ranjan, P., et al., Orange-fleshed cucumber (Cucumis sativus var. sativus L.) germplasm from North-East India: agro-morphological, biochemical and evolutionary studies. Genetic Resources and Crop Evolution, 2019. 66(6): p. 1217-1230. doi.org/10.1007/s10722-01900778-6.

[25] Mubin, N.A., et al., Young and mature oil palm tree detection and counting using convolutional neural network deep learning method. International Journal of Remote Sensing, 2019. 40(19): p. 7500-7515. DOI: $10.1080 / 01431161.2019 .1569282$.

[26] Hussain Hassan, N.M. and A.A. Nashat, New effective techniques for automatic detection and classification of external olive fruits defects based on image processing techniques. Multidimensional Systems and Signal Processing, 2019. 30(2): p. 571-589. DOI: 10.1007/s11045-0180573-5.

[27] Ponce, J.M., et al., Automatic Counting and Individual Size and Mass Estimation of Olive-Fruits Through Computer Vision Techniques. IEEE Access, 2019. 7: p. 59451-59465.

[28] Ponce, J.M., A. Aquino, and J.M. Andújar, Olive-Fruit Variety Classification by Means of Image Processing and Convolutional Neural Networks. IEEE Access, 2019. 7: p. 147629-147641. doi: 10.1109/ACCESS.2019.2947160.

[29] Gonzalez-Fernandez, I., et al., A critical review on the use of artificial neural networks in olive oil production, characterization and authentication. Critical Reviews in Food Science and Nutrition, 2019. 59(12): p. 1913-1926. DOI: 10.1080/10408398.2018.1433628.

[30] Furferi, R., L. Governi, and Y. Volpe, ANN-based method for olive Ripening Index automatic prediction. Journal of Food Engineering, 2010. 101(3): p. 318-328. doi.org/10.1016/j.jfoodeng.2010.07.016.

[31] Aguilera Puerto, D., et al., Online system for the identification and classification of olive fruits for the olive oil production process. Journal of Food Measurement and Characterization, 2019. 13(1): p. 716-727. doi.org/10.1007/s11694-018-9984-0.

[32] Diaz, R., et al., Comparison of three algorithms in the classification of table olives by means of computer vision. Journal of Food Engineering, 2004. 61(1): p. 101-107. doi.org/10.1016/S0260-8774(03)00191-2.

[33] Hassan, H., A.A. El-Rahman, and M. Attia. Color properties of olive fruits during its maturity stages using image analysis. in AIP Conference Proceedings. 2011. AIP.

[34] Guzmán, E., et al., Determination of the olive maturity index of intact fruits using image analysis. Journal of Food Science and Technology, 2015. 52(3): p. 1462-1470. DOI.org/10.1007/s13197-013-1123-7.

[35] Mora, M., J. Aliaga, and C. Fredes. Olive Ripening Phase Estimation based on Neural Networks. in IX Congreso Argentino de AgroInformática (CAI 2017)-JAIIO 46-43 CLEI (Córdoba, 2017). 2017.

[36] Navarro Soto, J.S.M., S.; Martínez Gila, D.; Gómez Ortega, J.; Gámez García, J., Fast and Reliable Determination of Virgin Olive Oil Quality by Fruit Inspection Using Computer Vision. Sensors, 2018. 11(18). DOI.org/ 10.3390/s18113826.

[37] Pariente, E.S., et al., On-site images taken and processed to classify olives according to quality - The foundation of a high-grade olive oil. Postharvest Biology and Technology, 2018. 140: p. 60-66. doi.org/10.1016/j.postharvbio.2018.02.012. 
[38] Demuth, H.; Beale, M.H.; Hagan, M.T.: Neural Network ToolboxUser's Guide. The MathWorks, Inc., Natrick (2009).

[39] Alkhasawneh, M.S. and L.T. Tay, A Hybrid Intelligent System Integrating the Cascade Forward Neural Network with Elman Neural Network. Arabian Journal for Science and Engineering, 2018. 43(12): p. 6737-6749. doi.org/10.1007/s13369-017-2833-3.
[40] Gao, X.Z.; Gao, X.M.; Ovaska, S.J.: A modified Elman neural net-work model with application to dynamical systems identification.In: IEEE International Conference on Systems, Man, and Cyber-netics, 1996 (1996).

[41] Hassan, H., A. El-Rahman, and M. Attia, Color Properties Of Olive Fruits During Its Maturity Stages Using Image Analysis. AIP Conference Proceedings, 2011. 1380. 